\title{
Problema de localización de bodegas y ruteo de vehículos resuelto mediante el optimizador de hiena manchada
}

\author{
Wilmer D. Urango, Helman E. Hernández y Jorge M. López \\ Facultad de Ingenierías, Dpto. de Ingeniería Industrial, Universidad de Córdoba, Carrera 6 N 76 - 103, Montería, \\ Córdoba, Colombia. (correo-e: wurangonarvaez86@correo.unicordoba.edu.co; hhernandez@correo.unicordoba.edu.co; \\ jotalopez@correo.unicordoba.edu.co)
}

Recibido Ago. 19, 2019; Aceptado Oct. 5, 2019; Versión final Nov. 28, 2019, Publicado Abr. 2020

\section{Resumen}

El objetivo principal de la investigación fue determinar la eficiencia de la metaheurística conocida como optimizador de hiena manchada en un problema de localización de bodegas y ruteo de vehículos con depósitos y vehículos capacitados, catalogado como un problema NP-Duro (No determinístico polinomial). Se comparó los resultados obtenidos mediante el optimizador de hiena manchada para un conjunto de situaciones seleccionadas de la literatura con sus correspondientes valores óptimos hallados hasta la fecha. Se encontró que el optimizador de hiena manchada ofrece buenos resultados para una parte de las situaciones, quedando cerca del óptimo en algunas. Los mejores resultados fueron para las instancias coor $100 * 10-1$ y coor $50 * 5-1 b$ optimizando sus costos hasta en un $100 \%$. En efecto, se puede afirmar que el optimizador de hiena manchada es una metaheurística adecuada para resolver instancias grandes para un problema de localización de bodegas y ruteo de vehículos.

Palabras clave: problema de ruteo de vehículos; optimizador de hiena manchada; metaheurística; optimización; logística

\section{Capacitated location routing problem solved by using the spotted hyena optimizer}

\begin{abstract}
The main objective of the research is to determine the efficiency of the spotted hyena optimizer metaheuristic in solving the capacitated location routing problem, recognized as a NP-hard problem (non-deterministic polynomial-time hardness). The results obtained using the spotted hyena optimizer were compared with literature results with their corresponding optimal values available until today. It was found that the spotted hyena optimizer offers good results for a part of the set of instances, being near to the optimum value in some cases. The best results were found for the instances coor $100 * 10-1$ and coor $50 * 5-1 \mathrm{~b}$, optimizing the cost up to $100 \%$. In fact, it can be said that the spotted hyena optimizer is an adequate metaheuristic method when is used to solve big instances of the capacitated location routing problem.
\end{abstract}

Keywords: vehicle routing problem; spotted hyena optimizer; metaheuristic; optimization; logistic 


\section{INTRODUCCIÓN}

El problema de localización de bodegas y ruteo de vehículos con depósitos y vehículos capacitados (CLRP por sus siglas en inglés Capacitated Locations Routing Problem) es la combinación de dos problemas NPHARD, el problema de localización de instalaciones FLP (Falicily Locations Problem) y el problema de ruteo de vehículos VRP (vehicle Routing Problem) (Willmer et al., 2013); El CLRP es un problema logístico muy complejo que hace parte de la gestión de la cadena de suministros (Zare y Nadizadeh, 2013). Para definir bien este problema suponga que se tiene un conjunto de posibles ubicaciones de centros de distribución o depósitos y un conjunto de clientes con demanda conocida, ahora, el propósito del CLRP es elegir qué centros de distribución deben abrirse, posteriormente definir el grupo de clientes que se asignará a cada centro de distribución electo y por último se especifica el orden en que se visitarán dichos clientes. Todo lo anterior se lleva a cabo con el fin de minimizar los costos totales, que incluyen, el costo de distribución y el costo de ubicación de los depósitos (Ferreira y Santos, 2007).

Como ya se mencionó los CLRP son la fusión de un FLP y un VRP es por ello por lo que se puede decir que los CLRP son derivados de los VRP. La aplicación de los VRP en problemas reales es incontable, y trabajos como los de Gómez et al.,(2019) demuestran esta aplicabilidad, estos autores aplican un problema de ruteo en el sector salud en donde se necesita optimizar la recogida de medicamentos sobrantes y suministros en el mínimo tiempo posible. Para ello desarrollan un modelo matemático y lo resuelven de dos maneras distintas una con la metaheurística de enjambre de partículas PSO (particle swarm optimization) y la otra con la metaheurística de Recocido Simulado SA (Simulated annealing).

En la literatura, actualmente se encuentran varios métodos heurísticos y metaheurísticos capaces de hallar las mejores soluciones para este tipo de problemas, según Borges et al., (2016) el primero, es un método de búsqueda adaptativa aleatorizado codicioso, unido con una búsqueda local evolutiva, llamado GRAPS + ELS propuesto por Duhamel et al., (2010), es una hibridación de un GRAPS (Greedy Randomized Adaptive Search Procedure) con un ELS (Evolutionary Local Search), el cual toma ventajas de ambos métodos. Del primero toma el enfoque de arranque múltiple (Feo y Resende, 1995) y del segundo toma la eficiencia en el método de explotación (Wolf y Merz, 2007). Dentro de esta metaheurística se resalta la alternancia entre las vistas TSP (Traveling Salesman Problem) y las soluciones LRP (Location-Routing Problem), en donde la primera se transforma en la segunda luego de una división no trivial. Esta propuesta alcanzo a mejorar la mayoría de los resultados de tres conjuntos de instancias bien conocidos en la literatura.

Por otra parte, se encuentran los métodos propuestos por Ferreira y Queiroz, (2018), los cuales están basados en el recocido simulado SA (Simulated Annealing), la primera propuesta es llamada $\mathrm{H} 1$ y está compuesta por cuatro componentes: generar una solución inicial con la ayuda de un procedimiento codicioso, emplear 4 operadores de búsqueda de vecindario basados en movimientos tipo inserción e intercambio, perturbar la mejor solución encontrada, y por ultimo mejorar la solución encontrada por el método LinKernighan. El segundo método heurístico es llamado H2 y utiliza los mismos procedimientos anteriores excepto la tercera fase, esto lo propusieron los autores debido que este paso puede llevar mucho tiempo de ejecución. Al final de su estudio lograron demostrar que ambas propuestas son eficientes a la hora de resolver un CLRP.

En tercer lugar, se encuentra Willmer et al., (2013), el cual plantea un algoritmo heurístico hibrido de dos fases, en donde la primera fase se encarga de construir una solución factible inicial, y la segunda parte se encarga de diversificar y mejorar la solución inicial por medio de una búsqueda tabú granular modificada GTS (por sus siglas en ingles Granular Tabu Search), añadido a esto el algoritmo también cuenta con un proceso de perturbación aleatorio, el cual es utilizado por los autores para evitar los óptimos locales. Los resultados del estudio demostraron la efectividad del algoritmo propuesto, además, también lograron evidenciar que este algoritmo puede ampliarse a problemas tales como, problema de enrutamiento de ubicación periódico PLRP (Periodic Location-Routing Problem), el problema de enrutamiento de varios vehículos en el depósito (MDVRP por sus siglas en inglés, Multi Depot Vehicle Routing Problem), entre otras variaciones del CLRP.

Otra metaheurística que demuestra gran rendimiento es llamada Granular Variable Tabú Neighborhood Search (GVTNS) propuesta por Escobar et al., (2014), la cual propone una búsqueda tabú granular GTS (Granular Tabu Search) combinada dentro de una búsqueda de vecindad variable VNS (Variable Neighborhood Search), dicho método fue capaz de mejorar 5 de las instancias escogidas. Esta combinación asegura que la primera parte controle los cambios de vecindario y que la segunda parte guie el proceso usando distintas estructuras de vecindad tales como la técnica de inserción, intercambio, two-opt, doble inserción y por último doble intercambio. También, propuestas como la de Hemmelmayr y Gabriel (2012) encontraron buenas soluciones mejorando un porcentaje considerable de conjuntos de instancias de referencia, en este caso esto fue logrado por la eficiencia y la sencillez de la modelación matemática propuesta, la cual fue aplicada a un problema 2EVRP y un LRP, añadido a esto se suma la heurística utilizada, ALNS (Adaptive Large Neighborhood Search), la cual es combinada utilizando varios operadores ya existentes y otros que son propuestos por los autores para el problema en específico. 
Otra de las mejores propuestas encontradas en la literatura la ofrece Ting y Chen, (2013), el cual muestra un Algoritmo de Optimización de Colonia de Hormigas MACO (Multiple Ant Colony Optimization Algorithm) el cual descompone el CLRP en sus partes originales, es decir, en un Problema de Localización de Instalaciones (FLP) y un Problema de Ruteo de Vehículos (VRP) y los analiza de manera escalonada empezando con el FLP y luego, con respecto a este, encuentra las mejores rutas (análisis de VRP), este método logro actualizar algunas de las mejores soluciones del conjunto de instancias seleccionados y quedó cerca del óptimo en otras.

Continuando con la descripción de las mejores metaheurísticas diseñadas en la actualidad no se puede dejar de hablar del trabajo de Yu et al.(2019) el cual propone un algoritmo genético hibrido HGA (Hybrid Genetic Algorithm) que resuelve un CLRP con restricciones de capacidad más estrictas y es capaz de tener en cuenta el espacio de solución factible y no factible, esta propuesta fue validada con dos conjuntos de instancias, un conjunto de instancias clásicas y un conjunto de instancias reales en donde se obtuvieron buenos resultados, incluso se actualizaron algunos valores que se creían óptimos, se puede decir que esta investigación aporta más veracidad a la hora de analizar un problema de CLRP real.

Es claro afirmar que las metaheurísticas alcanzan buenas soluciones, pero no son las únicas en lograr buenos resultados, Pekel Y Soner, (2019) diseñaron un algoritmo heurístico que combina la Búsqueda de Vecindad Variable (VNS) y la Búsqueda Local Evolutiva (ELS). En esta investigación se considera las demandas de los clientes y los tiempos de viaje entre clientes y depósitos como variables difusas y deterministas, respectivamente, los tamaños de flota se consideran homogéneos y heterogéneos simultáneamente. El algoritmo propuesto se fundamenta en cuatro etapas fundamentales las cuales alcanzan a resolver de manera eficiente un estudio de caso, que tiene cincuenta y siete clientes y cinco puntos de distribución, efectivamente se resuelve en un tiempo razonable, este algoritmo tiene por nombre algoritmo VNSxELS.

Por otra parte, el Optimizador de hiena manchada SHO, por sus siglas en inglés Spotted Hyena Optimizer propuesto por Dhiman y Kumar, (2017), hasta la fecha de esta investigación, es una metaheurística nunca antes utilizada en problemas de optimización como lo es CLRP, solo se ha utilizado en funciones de prueba. EI SHO está basado en el comportamiento colaborativo y relación social que poseen las hienas manchadas, está constituido principalmente por tres fases: buscar e identificar la presa, rodearla y por último atacarla. En base a lo anterior el algoritmo demostró ser un procedimiento aceptable para ser usado como método de resolución de problemas complejos, esto se demostró realizando una comparación con otras metaheurísticas ya encontradas en la literatura, dicha comparación se realizó con 29 funciones bien conocidas. Añadido a lo anterior los autores resolvieron 5 problemas de ingeniería en los cuales comprobaron con más certeza la eficiencia del $\mathrm{SHO}$.

El presente artículo tiene como objetivo demostrar la eficiencia de la metaheurística Optimizador de Hiena Manchada en un Problema de CLRP, en donde los vehículos son de flota homogénea y se encuentran capacitados al igual que los posibles centros de distribución, además la demanda se toma como un dato conocido. El problema se resuelve cuando se decide que depósitos abrir y en qué orden se visitaran los clientes que se asignan a cada deposito.

Con el fin de mejorar los resultados encontrados en la literatura y cumplir con el objetivo propuesto se aplica este nuevo método que al final arroja buenos resultados, logrando actualizar el valor óptimo de dos de las quince instancias seleccionadas. Dichas instancias corresponden a coor $100^{*} 10-1$ y coor50*5-1b. En la primera se obtuvo un valor de $\$ 30236,000$ para la función objetivo y en la segunda se obtuvo un valor de $\$$ 38576,400 , cabe resaltar que los valores para estas instancias en la literatura son de $\$ 258242,640$ y de $\$ 61595,220$ respectivamente, lo que significa que hay una mejora hasta en un $100 \%$ en estas dos instancias. Para alcanzar el objetivo propuesto el documento se estructura de la siguiente manera. Metodología, en este apartado se describe a detalle cómo se realizó la investigación y como se llegó a los resultados; Resultados, en esta se muestran los principales resultados de la investigación y por último se muestran las Conclusiones en donde se describe el análisis de los resultados y se plantean los posibles trabajos futuros.

\section{METODOLOGÍA}

En esta sección se describe el procedimiento que se llevó a cabo para realizar la investigación y está conformando por seis subsecciones: 1) modelo matemático; 2) aplicación del optimizador de hiena manchada; 3) Mejora de la ruta seleccionada; 4) instancias, 5) cálculo del Gap y 5) experimentos computacionales.

\section{Modelo matemático}

En este trabajo se analiza el CLRP desde un grafo dirigido con aristas con carga $G(V, E, C)$. En donde $V$ es el conjunto de vértices y está integrado por dos subconjuntos $I$, $J$, donde $I$ posee $m$ posibles localizaciones de depósitos; $J$ es igual al conjunto $\mathrm{V}$ menos el conjunto $/ \mathrm{y}$ posee $n$ posibles clientes. Además, los costos de las aristas $x(i, j)$ están representados por $C(i, j) ; W(i)$ es la capacidad del depósito $i \in I$ y $O(i)$ es el costo de apertura asociado; $d(j)$ es la demanda del cliente $j \in J ; K$ representa la flota de vehículos homogéneos de 
capacidad $Q$ y pose un costo fijo $F$; el número de vehículos utilizados es considerada una variable de decisión (Prins y Prodhon, 2006); el costo total está conformado por el costo fijo de los vehículos, costo de los vehículos por arista y el costo de apertura de los depósitos.

Añadido a lo anterior, en este trabajo se tienen en cuenta las siguientes restricciones: 1) Cada cliente es visitado solo una vez; 2) Cada vehículo debe salir y entrar del mismo depósito; 3) La suma de la demanda asignada a un depósito no debe exceder la capacidad del mismo; 4) La suma de la demanda de los clientes asignada a un vehículo no puede exceder a la capacidad de este; 5) El número de vehículos es una variable de decisión. Así, el modelo matemático utilizado propuesto por Ferdi y Layeb, (2018) se describe a continuación:

$\operatorname{Min} \mathrm{Z}=\sum_{\mathrm{i} \in \mathrm{l}} \mathrm{o}_{\mathrm{i}} \mathrm{y}_{\mathrm{i}}+\sum_{\mathrm{i} \in \mathrm{V}} \sum_{\mathrm{j} \in \mathrm{V}} \sum_{\mathrm{k} \in \mathrm{K}} \mathrm{c}_{\mathrm{ij}} \mathrm{x}_{\mathrm{ijk}}+\sum_{\mathrm{k} \in \mathrm{K}} \sum_{\mathrm{i} \in \mathrm{l}} \sum_{\mathrm{j} \in J} F \mathrm{x}_{\mathrm{ijk}}$

$\sum_{k \in K} \sum_{i \in V} x_{i j k}=1 \quad \forall j \in J$

$\sum_{k \in K} \sum_{i \in V} d_{i} x_{i j k} \leq Q \quad \forall k \in K$

$\sum_{j \in V} x_{i j k}-\sum_{j \in J} x_{i j k}=0 \quad \forall k \in K, \forall i \in V$

$\sum_{i \in l} \sum_{j \in J} x_{i j k} \leq 1 \quad \forall k \in K$

$\sum_{i \in S} \sum_{j \in S} x_{i j k} \leq|S|-1 \quad \forall S \subseteq J, \forall k \in K$

$\sum_{u \in J} x_{i u k}+\sum_{u \in V \backslash\{J\}} x_{i u k} \leq 1+f_{i j} \quad \forall i \in I, \forall i \in J, \forall k \in K$

$\sum_{j \in J} d_{i} f_{i j} \leq W_{i} y_{i} \quad \forall i \in l$

$\mathrm{x}_{\mathrm{ijk}} \in\{0,1\} \forall \mathrm{i} \in \mathrm{I}, \forall \mathrm{j} \in \mathrm{J}, \forall \mathrm{k} \in \mathrm{K}$

$y_{i} \in\{0,1\} \forall i \in I$

$f_{i j} \in\{0,1\} \forall i \in I, \forall j \in J$

En la ecuación (1) se muestra la función objetivo como la suma de los costos totales; la restricción (2) garantiza que cada cliente sea visitado por un solo vehículo; la restricción (3) y (8) representan la capacidad de los vehículos y de los depósitos respectivamente; las ecuaciones (4) y (5) garantizan la continuidad de la ruta y el retorno del vehículo al depósito; la ecuación (6) garantiza la eliminación de subtours; la ecuación (7) indica que un cliente puede ser asignado a un depósito, si éste último está abierto y existe una ruta que los asocia; por ultimo las ecuaciones (9), (10) y (11) representan las variables de decisión booleanas. 


\section{Aplicación del Optimizador de Hiena Manchada}

En el grafico 8 puede observarse el comportamiento completo de esta metaheurística. Cabe resaltar que El $\mathrm{SHO}$ está basado en tres pasos, como se detalla en lo que sigue.

\section{Primer paso}

Identificación de la presa; una vez se evalúe cada una de las soluciones generadas en cada iteración se escogerá como presa aquella que tenga el valor objetivo más bajo, debido a que se está minimizando costos. Por ejemplo, suponga que la figura 1 representa un conjunto de soluciones para cuatro depósitos y nueve clientes, en donde, las primeras cuatro columnas (color gris) representan los depósitos potenciales, las siguientes cuatro columnas (color azul) toman el valor de "1" si su correspondiente deposito es abierto y el valor de "0" en caso contrario, las siguientes nueve columnas (color naranja) representan los clientes a visitar, la última columna (color verde) representa el valor objetivo de cada solución. Note que el valor "-1" es utilizado para separar la asignación de los clientes a cada deposito. Así, para la primera fila se tiene que, se abrirán los depósitos "12" y "11", además que al depósito "12" se le asignarán lo clientes "2", "5", "3" y al depósito "11" se le asignaran los clientes "4","1","9","7","8" y "6". Explicado lo anterior, observe que el valor objetivo más bajo lo ofrece la solución correspondiente a la fila tres, por lo que esta se toma como la "presa", las demás quedan como agentes de búsqueda (hienas).

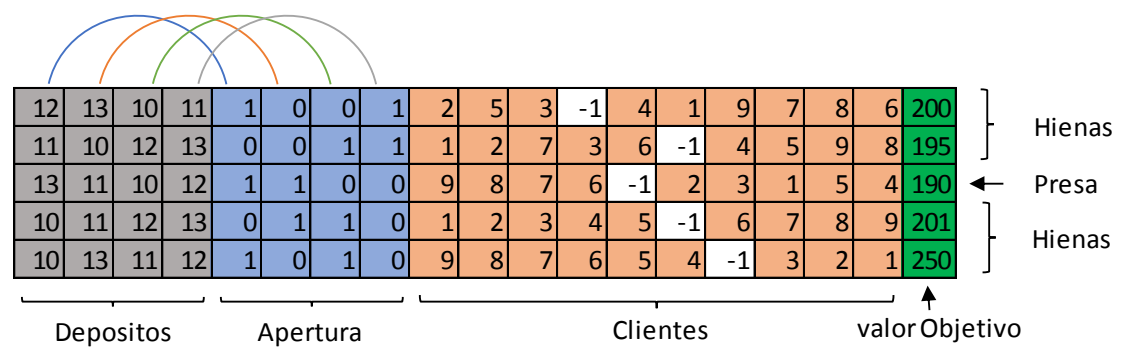

Fig. 1: Escoger presa

Para un mejor entendimiento, note que la presa sería la solución representada por la fila número 3 , la cual representa el valor más bajo. Si está maximizando la función objetivo, la presa corresponderá al valor más alto en la posición correspondiente a la función objetivo, en caso de estar minimizando se escogerá el menor valor para la función objetivo.

\section{Segundo paso}

Ataque de las hienas a la presa, en este caso, las hienas serán el resto de las soluciones seguidas del resultado de la presa del conjunto de soluciones generado; el ataque se realiza mediante un proceso de semejanza. Cuando se ataca, en realidad la hiena se está acercando a la presa lo que se representa mediante un cambio parcial del código de la hiena atacante, en otras palabras, se cambia un porcentaje de elementos del código del vector de la hiena atacante y se reemplaza por los elementos de la presa, esto garantiza un acercamiento al valor ofrecido por la presa. Cabe resaltar que el porcentaje mencionado se genera de manera aleatoria, además las posiciones de los elementos del vector a cambiar también se generan de manera aleatoria. Por ejemplo: suponga que la figura 2 representa la presa seguida de la hiena. La hiena es también llamada agente de búsqueda.

\begin{tabular}{|l|l|l|l|l|l|l|l|l|l|l|l|r|r|r|r|r|r|r|}
\hline 13 & 11 & 10 & 12 & 1 & 1 & 0 & 0 & 9 & 8 & 7 & 6 & -1 & 2 & 3 & 1 & 5 & 4 & 190 \\
\hline 10 & 11 & 12 & 13 & 0 & 1 & 1 & 0 & 1 & 2 & 3 & 4 & 5 & -1 & 6 & 7 & 8 & 9 & 201 \\
\hline
\end{tabular}

Fig. 2: Presa y agente de búsqueda

Este paso está basado en la siguiente ecuación:

$\mathrm{E}=\llbracket \mathrm{r}^{*}(\mathrm{EL}) \rrbracket+1$

Donde, $E$ es el número de elementos que se modificarán del vector en cada tipo de elemento, dependiendo del tipo de ataque (parcial o total, ver figura 8), por ejemplo si el ataque es parcial solo se intercambiaran los elementos correspondientes a los clientes, si el ataque es total se intercambiaran los elementos de los depósitos, de la apertura de los depósitos y de los clientes entre los vectores presa y hiena; $r$ es un número aleatorio que se genera en cada interacción, es análogo a la probabilidad de cruce o mutación en un Algoritmos Genético; y $E L$ es el número de elementos del vector analizado, note que este valor también depende del tipo de ataque, por ejemplo si el ataque es parcial el número de elementos del vector será igual al número de clientes. 
Ahora, supongamos que para una iteración dada $r=0.1$, se está realizando un ataque parcial por lo que $E L=9$, luego el número de elementos del vector a reemplazar es:

$\mathrm{E}=\llbracket 0,1^{*} 9 \rrbracket+1=1$

Notemos que el resultado para $\mathrm{E}$ es uno, por tanto, el agente de búsqueda solo intercambiara un elemento de la sección correspondiente a los clientes por un elemento de la presa en la misma posición. La ubicación de intercambio también se genera de manera aleatoria. ver figura 3. Tenga en cuenta que estos pasos se están realizando cumpliendo las restricciones de capacidad de los vehículos, capacidad de los depósitos y demanda de los clientes.

El paso anterior garantiza que el agente de búsqueda se vaya acercando a la presa o análogamente que el valor objetivo encontrado por el agente de búsqueda se acerque al valor objetivo de la presa en el espacio de soluciones.

\begin{tabular}{|c|c|c|c|c|c|c|c|c|c|c|c|c|c|c|c|c|c|c|}
\hline 13 & 11 & 10 & 12 & 1 & 1 & 0 & 0 & 9 & 8 & 7 & 6 & -1 & 2 & 3 & 1 & 5 & 4 & 190 \\
\hline 10 & 11 & 12 & 13 & 0 & 1 & 1 & 0 & 1 & 2 & 3 & 4 & 5 & -1 & 6 & 7 & 8 & 9 & 201 \\
\hline 13 & 11 & 10 & 12 & 1 & 1 & 0 & 0 & 9 & 8 & 7 & 6 & -1 & 2 & 3 & 1 & 5 & 4 & 190 \\
\hline 10 & 11 & 12 & 13 & 0 & 1 & 1 & 0 & 1 & 2 & 7 & 4 & 5 & \begin{tabular}{|c|} 
\\
\end{tabular} & 6 & 3 & 8 & 9 & 200 \\
\hline
\end{tabular}

Fig. 3: Cambio de elementos

Observe que, según la figura 3 se escoge de manera aleatoria la posición 11 del vector de la presa correspondiente al elemento "7", luego en la misma posición del vector hiena se coloca el elemento "7". Luego, para no generar soluciones no factibles, que en este caso se presentaría repitiendo los clientes al momento de realizar el intercambio, se toma el elemento que se acaba de reemplazar en este caso es " 3 " en el vector hiena y se coloca en la posición donde se encontraba el elemento "7", así la nueva solución no pierde factibilidad. Cabe resaltar que el fitness se recalcula con las nuevas posiciones de los valores. En el caso de un ataque total se realizaría el mismo procedimiento para cada tipo de elementos en el vector, es decir, se realizan los pasos para los elementos correspondientes a los depósitos, la apertura de los depósitos y los clientes.

\section{Tercer paso}

Por último, se encuentra el alejamiento de la presa, el cual es un movimiento utilizado para escapar de óptimos locales, y funciona, para este caso, de manera semejante al procedimiento anterior. En este caso los elementos que se van a asignar en las posiciones escogidas aleatoriamente de la hiena atacante se generan nuevamente de manera aleatoria, es decir, no se cambian los elementos por los de la presa como en el paso anterior.

\section{Mejora de la ruta seleccionada}

Durante el proceso de selección de la mejor ruta, se implementó una forma sencilla y eficiente de mejorar dicha ruta, esta mejora está basada en un análisis geométrico de ángulos en el plano, es propuesto por los autores de esta investigación y lo que busca es reducir el camino que tomarán los vehículos evitando el cruce o repetición de recorridos en la misma ruta.

\section{Primer paso}

Se selecciona de manera aleatoria un depósito; en este paso, el algoritmo ha decidido que depósito ha de abrirse, y lo hace tal como se muestra en la figura 1, de manera gráfica observe la figura 4;

\section{Segundo paso}

Se identifican los clientes que se le asignarán a una ruta del depósito escogido, (círculos de color verde en la figura 4 ) y se coloca dicho depósito como origen de un plano, Ver figura 5. Note que la codificación de este paso se muestra en la figura 1 en donde el valor de "-1" divide los clientes que se asignarán a cada deposito. 


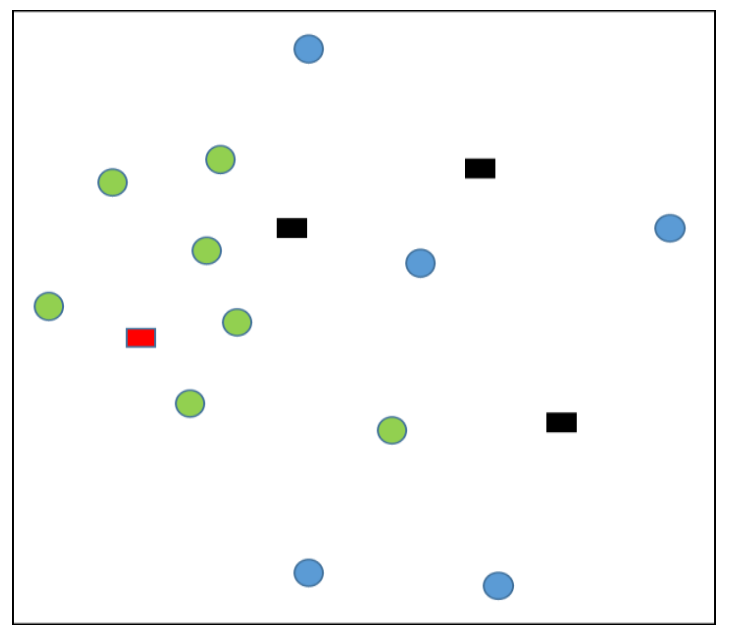

Fig. 4: Seleccionar depósito

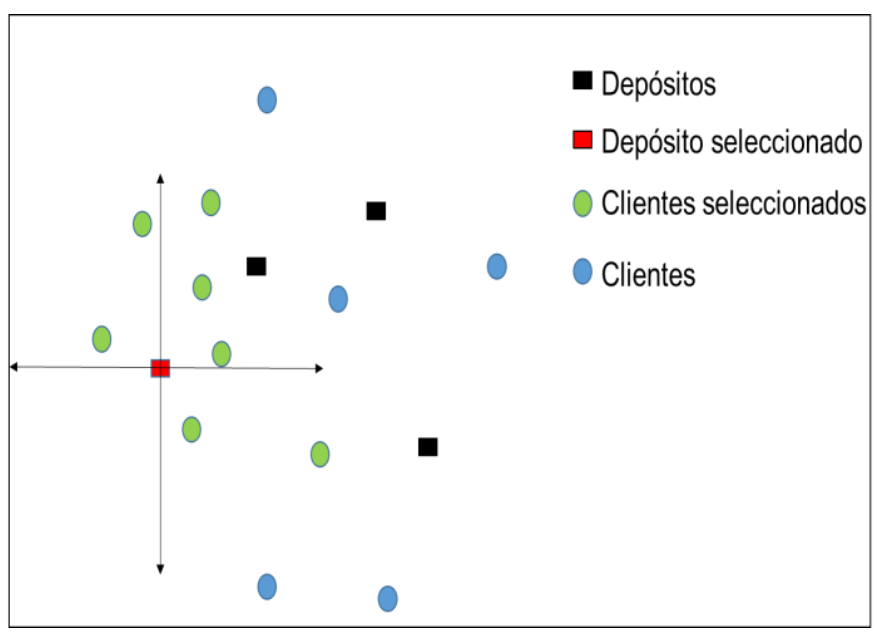

Fig. 5: Asignación de Clientes al Depósito

\section{Tercer paso}

Ya aquí, se determina el Angulo que le corresponde a cada cliente en su ubicación en el plano. El ángulo mencionado se halla con la ayuda de la fórmula:

$\theta_{i}=\tan ^{-1}\left(\left|\frac{y_{j}-y_{i}}{x_{j}-x_{i}}\right|\right)$

En donde $\theta(i)$ representa el ángulo del cliente $i ; y(j)$ es la coordenada en $y$ del depósito $j ; x(j)$ es la coordenada en $x$ del depósito $j ; y(i)$ es la coordenada en $y$ del depósito $i ; x(i)$ es la coordenada en $x$ del depósito $i$. Cabe resaltar que la ecuación (12) debe aplicarse teniendo en cuenta el cuadrante en que se encuentra el cliente según el depósito al cual está asignado.

\section{Cuarto paso}

Por último, se ordenan de menor a mayor los ángulos correspondientes a cada cliente (ver figura 6) y ese será el orden de visita a los clientes, (ver figura 7). El proceso completo del SHO se observa en la figura 8.

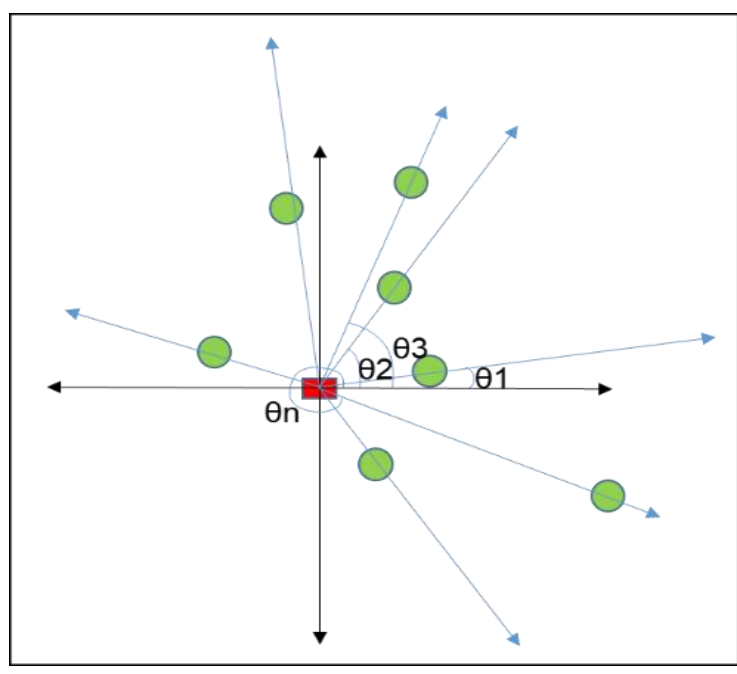

Fig. 6: Clientes con su ángulo respetivo

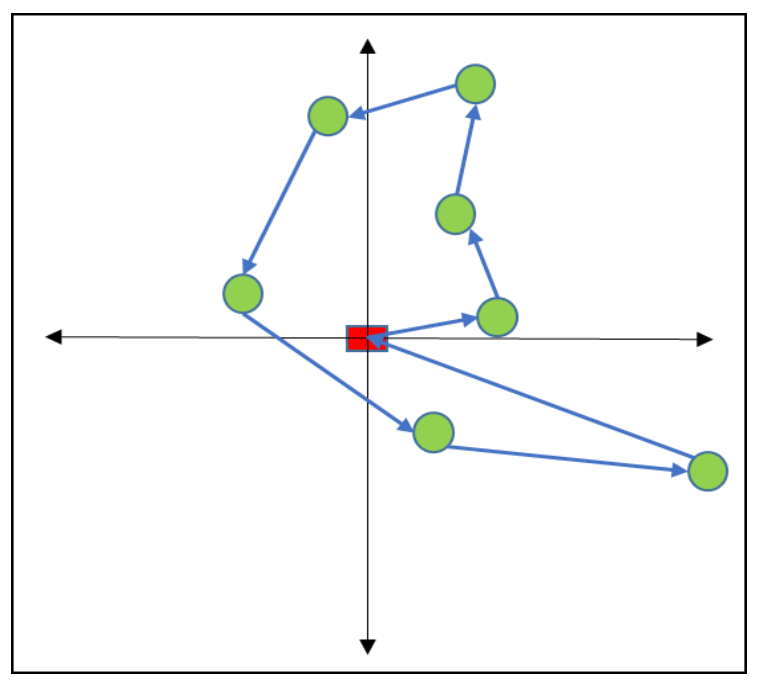

Fig. 7: Ruta mejorada

\section{Instancias}

Para demostrar los resultados de este trabajo se escogieron 15 instancias propuestas por Barreto S. (2004). Dichas instancias son las que se muestran en la Tabla 1. 


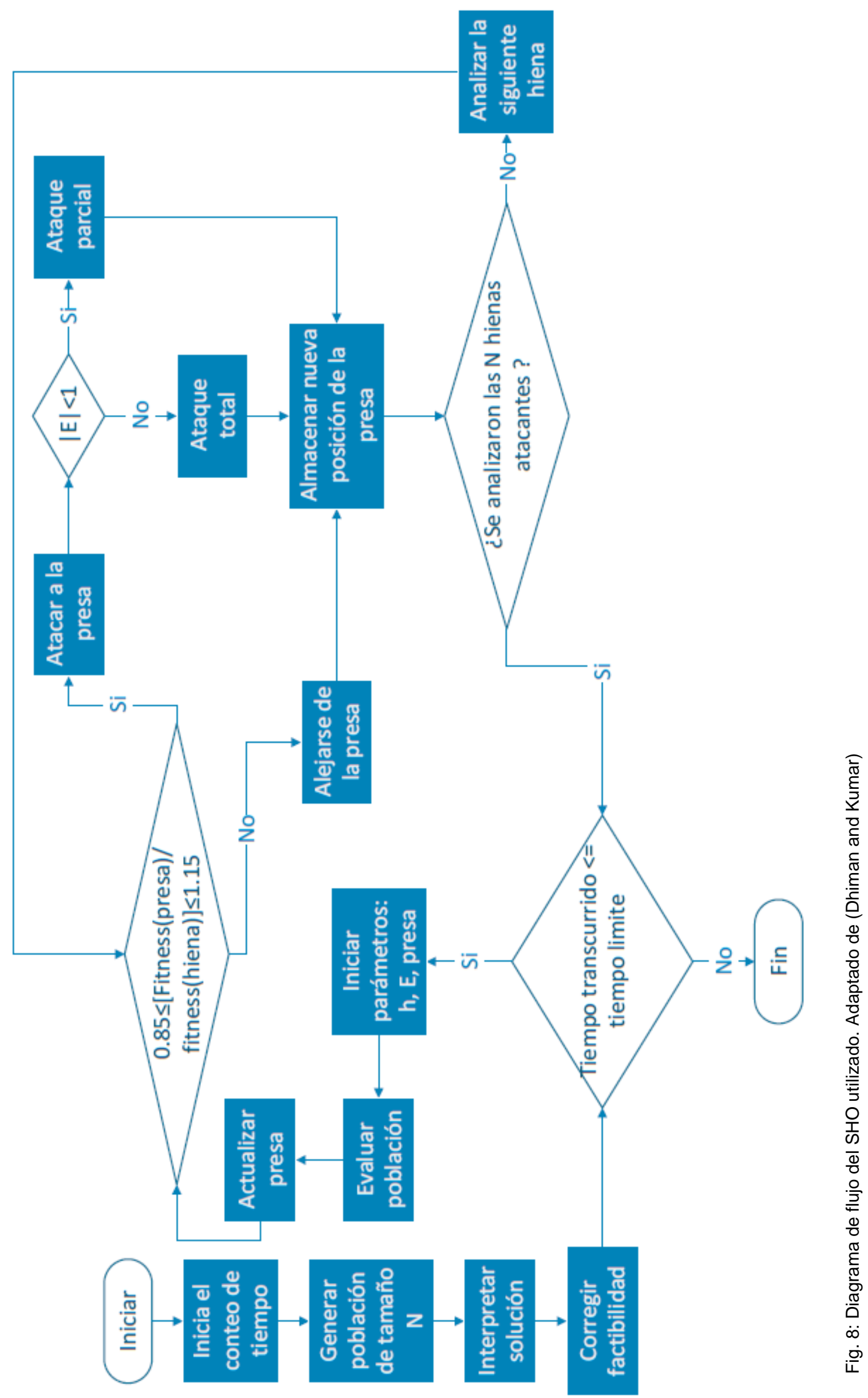


Tabla 1: Instancias

\begin{tabular}{|c|c|c|c|}
\hline $\begin{array}{l}\text { Nombre } \\
\text { instancia }\end{array}$ & $\begin{array}{c}\text { Número } \\
\text { de } \\
\text { clientes }\end{array}$ & $\begin{array}{c}\text { Cantidad } \\
\text { de } \\
\text { depósitos }\end{array}$ & Fuente \\
\hline coor50-5 & 50 & 5 & \multirow{6}{*}{$\begin{array}{c}\text { (Prins et } \\
\text { al., } \\
\text { 2006) }\end{array}$} \\
\hline coor20-5 & 20 & 5 & \\
\hline coor $100 * 10-1$ & 100 & 10 & \\
\hline $\operatorname{coor} 50 * 5-1 b$ & 50 & 5 & \\
\hline coor50-5 & 50 & 5 & \\
\hline coor100-5 & 100 & 5 & \\
\hline coorGaspelle & 21 & 5 & \multirow{2}{*}{$\begin{array}{c}\text { (Barreto, } \\
2004)\end{array}$} \\
\hline coorGaspelle2 & 22 & 5 & \\
\hline
\end{tabular}

\begin{tabular}{|c|c|c|c|}
\hline $\begin{array}{l}\text { Nombre } \\
\text { instancia }\end{array}$ & $\begin{array}{c}\text { Número } \\
\text { de } \\
\text { clientes }\end{array}$ & $\begin{array}{c}\text { Cantidad } \\
\text { de } \\
\text { depósitos }\end{array}$ & Fuente \\
\hline coorGaspelle3 & 29 & 5 & \multirow{7}{*}{$\begin{array}{c}\text { (Barreto, } \\
\text { 2004) }\end{array}$} \\
\hline coorGaspelle4 & 32 & 5 & \\
\hline coorMin27 & 27 & 5 & \\
\hline coorGaspelle6 & 36 & 5 & \\
\hline coorChrist75 & 75 & 10 & \\
\hline coorChrist100 & 100 & 10 & \\
\hline coorDas50 & 150 & 10 & \\
\hline * & * & * & * \\
\hline
\end{tabular}

\section{Cálculo del Gap}

En esta investigación se analizaron los resultados por medio del Gap, que para este caso representa la brecha existente entre el promedio de los resultados por cada instancia y el correspondiente resultado encontrado en la literatura. En otras palabras, el Gap se calculó hallando el promedio de los resultados obtenidos por el $\mathrm{SHO}$ por las réplicas de cada instancia con respecto a los valores encontrados en la literatura. Para este caso, el Gap es el factor que representa los resultados obtenidos en esta investigación en función del valor óptimo encontrado en la literatura y es calculado como se muestra en la ecuación (13):

$$
\text { Gap }=\frac{\text { promedio de los resultados encontrado por el SHO }- \text { mejor resultado de la literatura }}{\text { mejor resultado de la literatura }}
$$

\section{Experimentos computacionales}

El experimento se inició declarando los parámetros que se utilizarían para llevar a cabo el experimento, estos fueron los siguientes: 1) Agentes de búsqueda $N=70 ; 2$ ) parámetro de control h $\epsilon$ [5 0] y el otro parámetro que ofrece la metaheurística original es el número de iteraciones, pero en esta investigación se optó por asignar un tiempo límite de 120 minutos en vez de utilizar un cierto número de iteraciones. Luego de escoger los parámetros se ejecutó el experimento en 4 equipos idénticos, realizando 15 réplicas por cada una de las 15 instancias seleccionadas. Además, a cada réplica se le asignó un tiempo límite de 120 minutos. En total se realizaron 225 réplicas las cuales fueron distribuidas proporcionalmente y aleatoriamente en los 4 equipos.

Cabe resaltar que para cada una de las corridas del experimento se utilizó el software Python 3.5 con un tiempo límite de 120 minutos para cada una de las réplicas. Esto se realizó en cuatro computadoras con procesador Intel (R) Core (TM) i7-4790 CPU @ 3.60GHz, de 8 GB RAM y sistema operativo Windows 8.1 Pro de 64 bits. Añadido a lo anterior, se puede mencionar que se realizó un experimento simple en el cual se analizó el Gap y la desviación estándar de este para cada uno de los resultados de las instancias seleccionadas. Esto se hizo teniendo como referencia los mejores resultados obtenidos en la literatura para cada una de las instancias.

\section{RESULTADOS}

Cabe resaltar que no se describen resultados paso a paso como se explica en la sección anterior debido a que el algoritmo fue automatizado de tal manera que al iniciar el proceso solo queda esperar los resultados. Es por ello por lo que luego de que el algoritmo realizara todo ese proceso de manera interna se arrojaron los resultados que se muestran en la Tabla 2.

Tabla 2: Resultado del experimento (datos columna tres tomados de Classical Instances for LRP, 2010)

\begin{tabular}{|l|c|c|c|c|c|}
\hline Instancia & Mejor resultado por SHO & $\begin{array}{c}\text { Promedio de los resultados } \\
\text { encontrados por SHO }\end{array}$ & $\begin{array}{c}\text { Mejor resultado } \\
\text { en la literatura }\end{array}$ & Gap & Gap SD \\
\hline coorMin27 & 5982,000 & 6286,400 & 3062,000 & 1,053 & 0,069 \\
\hline coor20-5 & 69749,000 & 74381,867 & 54793,000 & 0,357 & 0,058 \\
\hline coorChrist75 & 2030,000 & 2163,200 & 798,700 & 1,708 & 0,096 \\
\hline coorChrist100 & 2050,000 & 2219,733 & 818,100 & 1,713 & 0,109 \\
\hline coor50-5 & 154886,000 & 168245,133 & 87109,640 & 0,931 & 0,087 \\
\hline
\end{tabular}


Tabla 2: (Continuación)

\begin{tabular}{|l|c|c|c|c|c|}
\hline coorDas50 & 141208,000 & 150410,800 & 43406,000 & 2,465 & 0,107 \\
\hline coorGaspelle & 766,000 & 839,467 & 424,900 & 0,975 & 0,078 \\
\hline coorGaspelle2 & 840,000 & 960,400 & 585,100 & 0,641 & 0,081 \\
\hline coorGaspelle3 & 922,000 & 1015,600 & 512,100 & 0,983 & 0,070 \\
\hline coorGaspelle4 & 982,000 & 1018,800 & 562,200 & 0,812 & 0,046 \\
\hline coor50-5 & 163319,000 & 177932,333 & 86055,010 & 1,067 & 0,066 \\
\hline coorGaspelle6 & 776,000 & 826,267 & 460,400 & 0,794 & 0,062 \\
\hline coor100*10-1 & 30236,000 & 33016,800 & 258242,640 & $-0,872$ & 0,004 \\
\hline coor50*5-1b & 38576,400 & 41531,000 & 61595,220 & $-0,325$ & 0,028 \\
\hline coor100-5 & 420996,000 & 456116,333 & 272082,370 & 0,676 & 0,060 \\
\hline & & & Promedio & 0,865 & 0,068 \\
\hline
\end{tabular}

Note que en la tabla 2 el campo "Gap" está calculado con respecto al campo "Promedio de los resultados encontrados por SHO" el cual representa el promedio de los resultados obtenidos por el SHO por cada replica por instancia. Para calcular el campo Gap SD, se calculó el Gap por cada replica por instancia y luego se halló la desviación estándar por cada conjunto de réplicas por instancia. El SHO muestra un Gap promedio de 0,865 el cual indica que en promedio los resultados obtenidos están representados por un $86,5 \%$ de los valores óptimos encontrados en la literatura, además poseen una desviación estándar de 0,068 el cual indica que los resultados en promedio están poco dispersos con respecto a los valores medios de los resultados.

\section{DISCUSION}

Ahora, según la Tabla 2 se puede afirmar que el SHO alcanza a mejorar los resultados de dos de las 15 instancias, note que el Gap para estas instancias es negativo lo que indica que el valor alcanzado por el SHO es menor al valor encontrado en la literatura. Las instancias que se logran optimizar son coor $100^{*} 10-1$ con 100 clientes y 10 posibles depósitos y la instancia coor 50 * 5 -1b con 50 clientes y 5 posibles depósitos, además se acerca al optimo en las instancias coor20-5 con 20 clientes y 5 posibles depósitos y queda lejos del optimo en las instancias restantes. Estas afirmaciones se basan en los resultados obtenidos en esta investigación en comparación con los resultados obtenidos por Barreto S. (2004).

Se puede decir que el algoritmo $\mathrm{SHO}$ es eficiente al momento de resolver instancias de gran tamaño como lo es la instancia coor $100^{*} 10-1$ la cual posee gran cantidad de clientes y depósitos; es regular para encontrar buenas soluciones en instancias pequeñas, aquellas de pocos clientes y depósitos; por último, se puede decir que SHO se queda corto a la hora de resolver instancias de mediano tamaño, con una cantidad considerable de clientes y depósitos. Estos resultados se ven reflejados en el Gap promedio de los resultados el cual arroja un valor de 0,865 , lo que indica, que los datos obtenidos por el $\mathrm{SHO}$ son en promedio el $86,5 \%$ de los valores hallados por Barreto S. (2004). Este valor es considerablemente alto teniendo en cuenta que la mayoría de las instancias son de mediano tamaño y como ya se mencionó el SHO es apto para instancias de gran calibre.

\section{CONCLUSIONES}

Luego de analizar y discutir los resultados obtenidos por el Optimizador de Hiena Manchada se puede concluir lo siguiente: 1) el SHO es eficiente a la hora de resolver instancias de gran tamaño en los problemas de localización de bodegas y ruteo de vehículos con depósitos y vehículos capacitados. 2) El SHO no es recomendable para resolver instancias medianas o pequeñas en problemas de CLRP ya que se queda corto en los resultados.

Por otra parte, cabe resaltar que en este estudio se tuvieron en cuenta métodos de búsqueda exploratoria análogos a los operadores del SHO, es decir, es posible mejorar aún más los resultados utilizando heurísticas especiales para ejecutar este tipo de búsquedas en el espacio de soluciones, en efecto, para trabajos futuros se implementaran dichas heurísticas con el fin de alcanzar mejores resultados.

\section{AGRADECIMIENTOS}

Los autores del presente trabajo agradecen a la Universidad de Córdoba de Colombia por el apoyo y asignación de recursos para llevar a cabo esta investigación.

\section{REFERENCIAS}

Barreto, S., Ferreira, C. y otros dos autores, Using Clustering Analysis in a Capacitated Location-Routing Problem, doi:10.1016/j.ejor.2005.06.074, European Journal of Operational Research, 179(3), 968-77, (2007). 
Barreto, S., Análise e Modelização de Problemas de localização-distribuição, Unpublished doctoral dissertation, University of Aveiro, Campus Universitário de Santiago, 3810-193, (2004).

Dhiman, G., y Kumar, V., Spotted Hyena Optimizer: A Novel Bio-Inspired Based Metaheuristic Technique for Engineering Applications, doi:10.1016/j.advengsoft.2017.05.014, Advances in Engineering Software, 114, 48-70, (2017).

Duhamel, C., Lacomme, P. y otros dos autores, A GRASP $\times$ ELS Approach for the Capacitated Location-Routing Problem, doi:10.1016/j.cor.2009.07.004, Computers \& Operations Research, 37(11), 1912-23, (2010).

Escobar, J.W., Linfati, R. y otros dos autores, A Granular Variable Tabu Neighborhood Search for the Capacitated Location-Routing Problem, doi:10.1016/j.trb.2014.05.014, Transportation Research Part B: Methodological, 67, 344-56, (2014).

Escobar, J.W., Linfati, R. y Toth, P., A Two-Phase Hybrid Heuristic Algorithm for the Capacitated Location-Routing Problem, doi:10.1016/j.cor.2012.05.008, Computers \& Operations Research, 40(1), 70-79, (2013).

Feo, T.A. y Resende, M.G., Greedy randomized adaptive search procedures, doi:10.1007/bf01096763, Journal of global optimization, 6(2), 109-133, (1995)

Ferdi, I., y Layeb, A., A GRASP Algorithm Based New Heuristic for the Capacitated Location Routing Problem, doi:10.1080/0952813X.2017.1421268, Journal of Experimental \& Theoretical Artificial Intelligence, 30(3), 369-87, (2018)

Ferreira, K.M., y Alves, T., Two Effective Simulated Annealing Algorithms for the Location-Routing Problem, doi:10.1016/j.asoc.2018.05.024, Applied Soft Computing, 70, 389-422, (2018).

Gómez, R.A., Salazar, F. y Rincon, N., Metaheurísticos Para Solución Del Problema de Ruteo En La Recuperación de Medicamentos Sobrantes y Suministros En Hospitales, doi:10.4067/S0718-07642019000200303, Información Tecnológica, 30(2), 303-14, (2019).

Hemmelmayr, V.C., Cordeau, J.F. y Crainic, T.G., An Adaptive Large Neighborhood Search Heuristic for Two-Echelon Vehicle Routing Problems Arising in City Logistics, doi:10.1016/j.cor.2012.04.007, Computers \& Operations Research, 39(12), 3215-28, (2012).

Lopes, R.B., Ferreira, C. y Santos, B.S., A Simple and Effective Evolutionary Algorithm for the Capacitated LocationRouting Problem, doi:10.1016/j.cor.2016.01.006, Computers \& Operations Research, 70, 155-62, (2016).

Pekel, E., y Soner, S., Solving Fuzzy Capacitated Location Routing Problem Using Hybrid Variable Neighborhood Search and Evolutionary Local Search, doi:10.1016/j.asoc.2019.105665, Applied Soft Computing, 83, 105665, (2019).

Prins, C., Prodhon, C. y Wolfler, R., Solving the Capacitated Location-Routing Problem by a GRASP Complemented by a Learning Process and a Path Relinking, doi:10.1007/s10288-006-0001-9, 4OR, 4(3), 221-38, (2006).

Ting, C.J. y Chen, C.H., A Multiple Ant Colony Optimization Algorithm for the Capacitated Location Routing Problem, doi:10.1016/j.ijpe.2012.06.011, International Journal of Production Economics, 141(1), 34-44, (2013).

Wolf, S. y Merz, P., Evolutionary Local Search for the Super-Peer Selection Problem and the p-Hub Median Problem, doi:10.1007/978-3-540-75514-2_1, Hybrid Metaheuristics, 4771, 1-15, (2007).

Yu, X., Zhou, Y. y Liu, X.F., A Novel Hybrid Genetic Algorithm for the Location Routing Problem with Tight Capacity Constraints, doi:10.1016/j.asoc.2019.105760, Applied Soft Computing, 85, 105760, (2019).

Zare, Y. y Nadizadeh, A., Using Greedy Clustering Method to Solve Capacitated Location-Routing Problem with Fuzzy Demands, doi:10.1016/j.ejor.2013.02.013, European Journal of Operational Research, 229(1), 75-84, (2013). 
